Density-matrix renormalization-group algorithms with nonorthogonal orbitals and nonHermitian operators, and applications to polyenes

Garnet Kin-Lic ChanTroy Van Voorhis

Citation: J. Chem. Phys. 122, 204101 (2005); doi: 10.1063/1.1899124

View online: http://dx.doi.org/10.1063/1.1899124

View Table of Contents: http://aip.scitation.org/toc/jcp/122/20

Published by the American Institute of Physics 


\title{
Density-matrix renormalization-group algorithms with nonorthogonal orbitals and non-Hermitian operators, and applications to polyenes
}

\author{
Garnet Kin-Lic Chan ${ }^{\text {a) }}$ \\ Department of Chemistry and Chemical Biology, Cornell University, Ithaca, New York 14853-1301 \\ Troy Van Voorhis \\ Department of Chemistry, Massachusetts Institute of Technology, Cambridge, Massachusetts 02139-4307
}

(Received 7 December 2004; accepted 9 March 2005; published online 20 May 2005)

\begin{abstract}
We describe the theory and implementation of two extensions to the density-matrix renormalization-group (DMRG) algorithm in quantum chemistry: (i) to work with an underlying nonorthogonal one-particle basis (using a biorthogonal formulation) and (ii) to use non-Hermitian and complex operators and complex wave functions, which occur naturally in biorthogonal formulations. Using these developments, we carry out ground-state calculations on ethene, butadiene, and hexatriene, in a polarized atomic-orbital basis. The description of correlation in these systems using a localized nonorthogonal basis is improved over molecular-orbital DMRG calculations, and comparable to or better than coupled-cluster calculations, although we encountered numerical problems associated with non-Hermiticity. We believe that the non-Hermitian DMRG algorithm may further become useful in conjunction with other non-Hermitian Hamiltonians, for example, similarity-transformed coupled-cluster Hamiltonians. (C) 2005 American Institute of Physics. [DOI: 10.1063/1.1899124]
\end{abstract}

\section{INTRODUCTION}

In this work we propose and implement two extensions to the density-matrix renormalisation-group (DMRG) algorithm of White, ${ }^{1,2}$ in the context of quantum chemistry. The DMRG is an ab initio algorithm that solves the manyparticle Schrödinger equation within an iteratively optimized subspace of fixed size $O(M)$ (where $M$ typically ranges from a few hundred to a few thousand). Unlike traditional subspace methods such as selected configuration interaction $(\mathrm{CI}),^{3}$ the DMRG subspace is not spanned by $O(M)$ Slater determinants, but rather, by complicated many-particle states, whose determinantal expansion is never computed, but for which we have generated enough information to obtain the matrix elements of the Hamiltonian. (A similar situation occurs in coupled-cluster (CC) theory, where we never directly manipulate the determinantal expansion of $e^{T}|0\rangle$.) The DMRG was designed to treat Hamiltonians of strongly correlated systems (e.g., the Heisenberg model of interacting spins ${ }^{4}$ ) which exhibit a macroscopic degeneracy of states in the Slater determinant basis, and thus cannot be studied with conventional active-space quantum chemical methods. In quantum chemistry, the DMRG has been developed in the work of White and Martin, ${ }^{5}$ Mitrushenkov et al. ${ }^{6,7}$ Chan and co-workers, ${ }^{8-11}$ and Legeza and co-workers. ${ }^{12-14}$ Our recent studies using the DMRG have produced new benchmark calculations, including a (numerically) exact solution of the Schrödinger equation for water in a triple-zeta plus double polarization (TZ2P) basis, ${ }^{10}$ and an all-electron benchmark binding curve for nitrogen dissociation at the correlation consistent polarized valence double zeta (cc-pVDZ) level. ${ }^{11}$

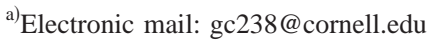

A key finding of these studies is that the DMRG provides a well-balanced description of the nondynamic correlation effects associated with bond-breaking processes, which can be attributed to the fact that unlike many methods, the DMRG is not biased towards a given reference and orbital occupancy, but instead treats all orbitals on an equal footing.

In the following, we will extend the existing quantum chemistry DMRG algorithm in two ways: (i) to work with an underlying nonorthogonal one-particle basis and (ii) to handle non-Hermitian and complex operators and complex wave functions. The central motivation for using nonorthogonal orbitals in the DMRG is to allow the use of localized nonorthogonal basis sets for calculations on extended systems, just as they are used in a number of low-scaling and linear-scaling correlation treatments. ${ }^{15,16}$ In extended chainlike systems, the DMRG algorithm can in principle achieve linear scaling. In a recent unpublished preprint, Mitrushenkov et al. already presented the theory behind a DMRG algorithm using nonorthogonal orbitals, although no implementation was given. ${ }^{17}$ In the current work, our formulation, although developed independently, is similar to the theory presented by those authors.

The extension of the DMRG to handle non-Hermitian and complex operators and complex wave functions arises naturally because we use a biorthogonal formalism to handle the nonorthogonal one-particle basis. (Mitrushenkov et al. also employ a biorthogonal formalism in Ref. 17.) NonHermitian DMRG algorithms are not new and have been used for some time, in the treatment of the thermodynamics of one-dimensional quantum systems. ${ }^{18-20}$ In a quantum chemistry setting, besides the application to nonorthogonal basis sets, a non-Hermitian DMRG is particularly interesting in the context of using the similarity-transformed Hamilto- 
nians $\bar{H}=e^{-T} H e^{T}$ that arise in CC theory, within the DMRG algorithm. This would offer a simple route to augmenting "active-space" DMRG calculations with a CC treatment of dynamical correlation, in the spirit of recent hybrid canonical diagonalization $^{21}$ and multireference coupled-cluster (MRCC) theories. ${ }^{22}$

Our presentation continues as follows. In Sec. I we describe the non-Hermitian DMRG algorithm for nonorthogonal orbitals, using a biorthogonal representation. The main challenges in working with a nonorthogonal, non-Hermitian DMRG are numerical in nature, and some of these challenges are described in Sec. I. In Sec. II we apply a preliminary implementation of this DMRG algorithm to study the electronic structure of the first three members of the polyene series: ethene, butadiene, and hexatriene. As the underlying nonorthogonal basis, we use the polarized atomic orbitals (PAOs) introduced by Lee and Head-Gordon, ${ }^{23}$ to form an active space. Our calculations correlate all electrons in this space, and therefore include the important $\sigma$ - $\pi$ correlations in these systems. We compare our new DMRG results to orthogonal orbital DMRG, second-order Møller-Plesset theory (MP2), and coupled-cluster singles and doubles (CCSD) calculations. In Sec. III, we summarize our findings and assess the potential of working with the nonorthogonal and non-Hermitian extensions of the DMRG algorithm.

\section{THEORY}

\section{A. Biorthogonal representation}

To construct a DMRG algorithm that uses an underlying nonorthogonal basis for both the bra and ket spaces is challenging at best, due to the necessity to compute the manyparticle overlap matrices in the many-particle DMRG basis. Instead, we choose to work in a biorthogonal representation, where the original nonorthogonal basis $\left|\phi_{i}\right\rangle$ is used only to span the ket space, and a dual set of vectors $\left\langle\phi^{i}\right|$ is chosen to span the bra space, such that (summation convention implied)

$$
\begin{aligned}
& \left\langle\phi^{i} \mid \phi_{j}\right\rangle=\delta_{j}^{i}, \\
& \left\langle\phi_{i} \mid \phi_{j}\right\rangle=S_{i j}, \\
& \left\langle\phi^{i}\right|=\left[S^{-1}\right]^{i j}\left\langle\phi_{j}\right| .
\end{aligned}
$$

Here we have used a tensor notation introduced by HeadGordon et al. $^{24}$

One might argue that in transforming to a biorthogonal representation-just as in transforming to a symmetrical orthogonal representation - one loses the benefits of the nonorthogonal basis, and that one could equally work well with the symmetrically orthogonalized basis from the start. The biorthogonal basis does provide certain advantages, most notably the fact that one only needs to perform a half-transform of the atom-centered two-electron integrals whereas a full transformation is required for symmetric orthogonalization. However, for the systems where DMRG is presently feasible, this has an exceedingly small effect on the overall cost. Our view is that biorthogonalization yields a basis which may or may not possess better qualities than the symmetrically orthogo- nalized basis for our calculation, but which is certainly different, and these differences are part of what we are investigating. We believe a similar point of view must motivate other explorations of biorthogonal formulations of correlation in nonorthogonal basis. ${ }^{16}$

Continuing, we can write down a set of creation and annihilation operators that construct the underlying oneparticle biorthogonal bras and kets,

$$
\begin{aligned}
& \langle-| a^{i}=\left\langle\phi^{i}\right|, \\
& a_{i}^{\dagger}|-\rangle=\left|\phi_{i}\right\rangle,
\end{aligned}
$$

and these obey the usual fermion commutation relations, e.g.,

$$
\begin{aligned}
& \left\{a^{\dagger i}, a_{j}\right\}=\delta_{j}^{i}, \\
& \left\{a^{i}, a_{j}\right\}=0 .
\end{aligned}
$$

Consequently, the quantum chemical Hamiltonian may be reexpressed in biorthogonal second quantization as

$$
H=t_{i}^{j} a^{\dagger i} a_{j}+\frac{1}{2} v_{i j}^{k l} a^{\dagger i} a^{\dagger j} a_{k} a_{l},
$$

where the matrix elements $t_{i}^{j}, v_{i j}^{k l}$ are obtained from the usual one-electron and two-electron matrix elements $t_{i j}, v_{i j k l}$ via

$$
\begin{aligned}
& t_{i}^{j}=t_{i k}\left[S^{-1}\right]^{k j}, \\
& v_{i j}^{k l}=v_{i j m n}\left[S^{-1}\right]^{m k}\left[S^{-1}\right]^{n l} .
\end{aligned}
$$

\section{B. The non-Hermitian DMRG algorithm}

Once we have the Hamiltonian in the biorthogonal representation (8), the DMRG algorithm may be formulated in a manner that parallels the usual Hermitian DMRG algorithm in an orthogonal basis. The essential difference is that because we are using different representations for the bra and ket spaces, all the operators involved in the DMRG are now non-Hermitian quantities. We shall refer the reader to Ref. 8 for the description of our Hermitian DMRG algorithm, and here we focus primarily on the new features arising from non-Hermiticity.

A DMRG algorithm consists of a sequence of sweeps through a predefined ordering of orbitals until convergence in some quantity (typically the sweep energy, i.e., the energy in the middle of the sweep) is reached. Each sweep consists of a set of iterations along the chain of orbitals. At every iteration in the sweep, the set of orbitals is divided into two blocks; system and environment; we shall avoid calling them left and right blocks as this might create confusion in the non-Hermitian formulation. A single iteration moves one spatial orbital, or equivalently two spin orbitals, from the environment to the system, thereby enlarging the system block and replacing the environment block with one which spans one fewer orbital.

All physical quantities can be expressed in terms of combinations of operators $O_{S}, O_{E}$ that act separately within the many-particle Fock spaces $\{S\},\{E\}$, spanned by the system and environment blocks of orbitals, respectively. As it is 
the system block which is growing at each iteration, the crucial step in the DMRG algorithm is to define how to transform the operators as expressed in the old system space $\{S\}$ [which is of dimension $O(M)]$ to the new renormalized $\{$ So $\}$ space of the same dimension $O(M)$ (here o denotes a spatial orbital, whose ket space $\{0\}$ is spanned by four states $|-\rangle,|\uparrow\rangle,|\downarrow\rangle,|\uparrow \downarrow\rangle)$.

This transformation may further be divided into three stages: (i) blocking, (ii) solving for the wave function of the desired state, and (iii) the many-particle basis transformation (decimation).

\section{Blocking}

Here we build representations of the operators we wish to compute in the tensor product space $\{S\} \otimes\{0\}$. So as long as we work within the biorthogonal bra, ket representation, then all operators in the enlarged space can be obtained, as in the Hermitian algorithm, via

$$
\left\langle\overline{s \mathrm{O}}\left|O^{S} O^{\mathrm{o}}\right| s^{\prime} \mathrm{o}^{\prime}\right\rangle=p\left\langle\bar{S}\left|O^{S}\right| s^{\prime}\right\rangle\left\langle\overline{\mathrm{o}}\left|O^{\mathrm{o}}\right| \mathrm{o}^{\prime}\right\rangle=p O_{s s^{\prime}}^{S} O_{\mathrm{oo}^{\prime}}^{\mathrm{o}},
$$

where $p$ is a phase $-1,+1$ arising from normal ordering in (11). (We have used bars to distinguish the bra states from the ket states of the same index.) However, the nonHermitian operators have reduced symmetry as compared to the operators in the Hermitian DMRG algorithm, e.g., $a^{\dagger i}$ $\neq\left(a_{i}\right)^{\dagger}$. Thus, we typically need to compute and store twice as many operator matrix elements as in the Hermitian DMRG algorithm. In addition, when working in the nonHermitian algorithm we will encounter complex matrix elements (in essence because after the decimation performed by the DMRG, the bra and ket spaces are no longer identical, and thus the Hamiltonian in this reduced space has complex eigenvalues and eigenvectors), and this leads to a further doubling of the storage and computing time requirements as compared to the usual Hermitian DMRG.

\section{Solving for the wave function}

Using the same formula (11), we can obtain a representation for the Hamiltonian in the full space of all the orbitals, $\{S o\} \otimes\{E\}$. Consequently, we can solve for one or more desired left, right eigenvectors $\langle\bar{\Psi}|,| \Psi\rangle$ of $H$ in the tensor product space

$$
\begin{aligned}
& \langle\bar{\Psi}|=\bar{c}_{\text {soe } e}\langle\overline{s \overline{\mathrm{O}} e}|, \\
& |\Psi\rangle=c_{\text {soe }}|s \mathrm{O} e\rangle,
\end{aligned}
$$

where $s o \in\{S \otimes \mathrm{o}\}, e \in\{E\}$, for the bra and ket spaces of the system and environment blocks, respectively. In addition to the need to solve for pairs of eigenvectors, the nonHermiticity of the Hamiltonian leads to the following differences: (i) the error in the energy is linear in the wavefunction error rather than quadratic, and (ii) since the Hamiltonian is solved in truncated bra and ket spaces that are not strictly identical (i.e., they do not have the same spanning basis), we will (and do) obtain complex eigenvalues and eigenfunctions.

Typically in the DMRG one uses an iterative solver, such as the Lanczos or Davidson algorithms, to obtain the desired eigenvalues and eigenvectors of $H$. We have implemented a non-Hermitian variant of the block Davidson-Liu algorithm. ${ }^{25}$ We find that the convergence characteristics of this algorithm are less favorable than in the Hermitian case. While part of this is due to the underlying quadratic convergence of the non-Hermitian Jacobi-Davidson algorithm as compared to the cubic convergence of the Hermitian algorithm, ${ }^{26}$ a more important factor in our case is the difficulty in finding a good preconditioner with which to compute the Davidson update vector. Due to the reduced localization of the one-particle basis for the bra space, transformation to the biorthogonal representation leaves the Hamiltonian less diagonally dominant than what we would typically encounter in the Hermitian algorithm. Consequently, a simple diagonal Davidson preconditioner is much less effective. We have also tried the variant of Olsen et al. of the Davidson algorithm, ${ }^{27}$ where the preconditioner is constructed by exactly inverting the Hamiltonian in a small subspace, but given the lack of a natural ordering of the complex diagonal matrix elements of the Hamiltonian, it is difficult to select a satisfactory starting subspace close to our desired roots. Thus, we have observed little improvement when employing the update scheme of Olsen et al. For all these reasons, in some difficult cases of convergence, we had to resort to explicit diagonalization of the Hamiltonian matrix in the full $\{S o\} \otimes\{E\}$ space. Given the large number of diagonalizations that need to be performed in each sweep [one for each step, and therefore $O(k)$ per sweep, where $k$ is the number of orbitals], and that the size of the full space $\{S o\} \otimes\{E\}$ is $O\left(16 M^{2}\right)$ (since typically $E$ is augmented with an exact $\operatorname{site}^{8}$ ), this currently limits practical calculations to $M \sim 50-100$, for which the dimension of the Hamiltonian matrix is roughly $5000 \times 5000$.

The cost of the non-Hermitian Davidson algorithm is eight times that of the Hermitian Davidson algorithm usually employed, due to the complex matrix multiply, and the need to solve for pairs of eigenvectors.

\section{Many-body basis transformation (decimation)}

In the decimation stage, we renormalize the blocked $\{S\} \otimes\{$ o $\}$ space of size $O(4 M)$ back down to $O(M)$, to form the space spanned by the next system block $S$ (that is one orbital larger) in the next sweep step. In the Hermitian DMRG algorithm, the renormalized space which best reproduces, in a least-squares sense, the current approximation to the target eigenvector $\Psi$ is spanned by the $M$ eigenvectors $\left|\psi_{m}\right\rangle$ of the reduced density matrix $\Gamma$ of the system with the largest eigenvalues $w_{m}$

$$
\Gamma=\operatorname{Tr}_{e}|\Psi\rangle\left\langle\Psi\left|=\sum_{m} w_{m}\right| \psi_{m}\right\rangle\left\langle\psi_{m}\right|
$$

and we transform our $\{S\} \otimes\{0\}$ to this truncated basis. In the case of the non-Hermitian algorithm, it is not entirely clear how to choose the optimal transformation, and there has been some debate in the literature. ${ }^{19,20}$ This is because given a biorthogonal pair of eigenvectors $\langle\bar{\Psi}|,| \Psi\rangle$, there is no single density-matrix operator from which we can select left and right eigenvectors to obtain optimal spaces in which to expand $\langle\bar{\Psi}|,| \Psi\rangle$, respectively. Instead, the analog of the 
Hermitian arguments shows that the optimal renormalized spaces in which to expand $\langle\bar{\Psi}|,| \Psi\rangle$ can be obtained from the left and right eigenvectors of the left and right density matrices $\bar{\Gamma}=\operatorname{Tr}_{e}|\bar{\Psi}\rangle\left\langle\bar{\Psi}\left|, \Gamma=\operatorname{Tr}_{e}\right| \Psi\right\rangle\langle\Psi|$ separately. These quantities are inaccessible in the biorthogonal representation, as we cannot obtain $|\bar{\Psi}\rangle$ from $\langle\bar{\Psi}|$, as this requires knowledge of the many-particle overlap matrix between the underlying many-particle bra and ket states in which $\bar{\Psi}$ and $\Psi$ are expanded. Consequently, the most natural choice for the density matrix is simply the asymmetric quantity (as used, for example, by Wang and Xiang ${ }^{19}$ and Mitrushenkov et al. ${ }^{17}$ )

$$
\begin{aligned}
\Gamma & =\operatorname{Tr}_{e}|\Psi\rangle\left\langle\bar{\Psi}\left|=\sum_{s s^{\prime} e} c_{s e}^{*} \bar{c}_{s e}\right| s\right\rangle\left\langle\bar{s}^{\prime}\right| \\
& =\sum_{s s^{\prime}} \gamma_{s s^{\prime}}|s\rangle\left\langle\bar{s}^{\prime}\left|=\sum_{m} w_{m}\right| \psi_{m}\right\rangle\left\langle\bar{\psi}_{m}\right| .
\end{aligned}
$$

If no truncation is performed in the DMRG algorithm, then Eq. (15) is simply the representation of the usual Hermitian density operator of the system in the biorthogonal basis. (As one would expect in that scenario, the operator that governs the selection of the optimal basis for a subsystem is independent of the choice of representations for the bra and ket spaces.) However, since we do renormalize our spaces in the DMRG, the eigenvalues $w_{m}$ of $\Gamma$ in Eq. (15) are complex, and thus have no simple ordering allowing us to determine the most important eigenvectors. Consequently, we have found it more convenient to choose a basis transformation to the eigenvectors of a corresponding "squared" density matrix, defined by

$$
\begin{aligned}
& \Gamma^{2}=\sum_{s s^{\prime}} d_{s s^{\prime}}|s\rangle\left\langle s^{\prime}\right|, \\
& d_{s s^{\prime}}=\sum_{s^{\prime \prime}}\left[\gamma^{\dagger}\right]_{s s^{\prime \prime}} \gamma_{s^{\prime \prime} s^{\prime}} .
\end{aligned}
$$

By construction, $d_{s s^{\prime}}$ is Hermitian and has positive eigenvalues, and we choose the renormalized $\{S\}$ to be spanned by the $M$ left, right eigenvectors of $\Gamma^{2}$ with the largest weights (eigenvalues). We then perform the linear transformation of operators in the old $\{S\} \otimes\{\circ\}$ representation to the new renormalized $\{S\}$ space spanned by these vectors.

Once all three stages of a single sweep step are complete, the algorithm proceeds to the next block configuration in the same way as in the usual Hermitian algorithm, eventually leading to a set of sweeps back and forth along the chain of orbitals. Typically, expectation values evaluated near the middle of the sweep are most accurate, as this corresponds to the largest size of the system and environment tensor product space $\{S\} \otimes\{E\}$. In our non-Hermitian DMRG calculations, we took the best ("sweep") energy in each sweep at the block configuration when the dimension of the space $\{S \circ\} \otimes\{E\}$ was largest, and sweeps were then repeated until convergence in this energy.

In summary, the use of complex matrix elements and non-Hermiticity leads to a memory cost four times that of the Hermitian algorithm $\left[O\left(M^{2} k^{2}\right)\right.$ per sweep $]$ and a computation cost roughly eight times that of the Hermitian algorithm $\left[O\left(M^{3} k^{3}\right)\right.$ per sweep], where $k$ is the total number of orbitals.

\section{THE POLYENE SERIES}

As a test of our non-Hermitian DMRG algorithm using nonorthogonal orbitals, we carried out preliminary groundstate calculations on the first three members of the polyene series: ethene $\left(\mathrm{C}_{2} \mathrm{H}_{4}\right)$, butadiene $\left(\mathrm{C}_{4} \mathrm{H}_{6}\right)$, and hexatriene $\left(\mathrm{C}_{6} \mathrm{H}_{8}\right)$. As $\sigma-\pi$ correlation effects are important in the spectrum of these systems, ${ }^{28,29}$ it is important to at least correlate both sets of electrons, and we have chosen to correlate all electrons, including the core electrons. As our basis, we use the PAO basis functions as introduced by Lee and Head-Gordon. ${ }^{23}$ The PAOs are a minimal basis set where each basis function $\eta_{i}^{A}$ is an atom-centered linear combination of functions from a larger secondary basis $\zeta_{j}$,

$$
\eta_{i}^{A}=\sum_{j} f_{i j}^{A} \zeta_{j}^{A} .
$$

The coefficients $f_{i j}^{A}$ are determined by minimizing the PAO basis Hartree-Fock energy with respect to $\zeta_{j}^{A}$ in Eq. (18). Although these basis sets have mainly been used in linearscaling calculations in extended molecules, ${ }^{23,30}$ as minimal basis sets, they function in this work primarily as a nonorthogonal basis for the $\sigma$ and $\pi$ active spaces in the polyenes.

Now, the PAOs are only defined up to a unitary transformation that mixes the orbitals on a given atom. As a result, the automatically generated PAOs do not correspond to lone pair orbitals, $s p^{2}$ bonding orbitals, etc. as one might expect based on chemical intuition. In order to obtain an intuitive set of PAOs (and to fix the ambiguity in their definition) we apply the natural hybrid ${ }^{31}(\mathrm{NH})$ transformation to the PAOs. Here, one considers the symmetrically orthogonalized PAO density matrix

$$
P^{\mu \nu}=\left[S^{-1 / 2}\right]^{\mu \lambda} \chi_{i \lambda} \chi_{i \nu}\left[S^{-1 / 2}\right]^{\nu \sigma},
$$

where $\chi_{i}$ are the molecular orbitals in the PAO basis and $\mu, \nu, \lambda$, and $\sigma$ index the raw PAOs. In order to obtain PAOs with the proper hybridization, one diagonalizes the different atom-atom blocks of $P$ separately. That is, one diagonalizes $P^{A A}, P^{A B}, P^{A C}$, etc. For each block, one selects the orbitals whose eigenvalues are near 2 (i.e., that are nearly doubly occupied). For the intraatomic block, this yields core and lone pair orbitals, while for interatomic blocks this gives the directed atomic hybrid orbitals (e.g., $p_{z}$ or $s p^{2}$ ). ${ }^{31}$ The final set of orbitals is then symmetrically orthogonalized on each atom to obtain the initial set of PAOs. This NH-PAO scheme results in a chemically motivated set of orbitals that are ordered by atom as we move from left to right along a chain.

The geometries of ethene, butadiene, and hexatriene were determined by optimization with $\mathrm{MP} 2 / 6-31 \mathrm{G}^{*}$ using Q-CHEM. ${ }^{32}$ PAOs were determined within Q-CHEM using a secondary $[5 s 4 p 3 d(\mathrm{C}) / 3 s 2 p(\mathrm{H})]$ basis; the $5 s 4 p 2 d$ basis (with spherical $d$ functions) is derived from the Dunning correlation consistent polarized valence quadruple zeta (ccpVQZ) basis ${ }^{33}$ by removing the $f$ and $g$ functions, and the $3 s 2 p$ basis on the hydrogens is derived from the Dunning correlation consistent polarized valence triple zeta (cc- 
TABLE I. Orbital orderings (for PAOs) used in calculations on $\mathrm{C}_{4} \mathrm{H}_{6}$. The indices refer to the atoms along $\mathrm{t}$ he $\mathrm{C}_{4} \mathrm{H}_{6}$ chain, which are labeled $H^{1} H^{2} C^{3} H^{4} C^{5} H^{6} C^{7} H^{8} C^{9} H^{10}$. PAOs are abbreviated as $h$ : hydrogen $1 s, l$ : carbon $s p^{2}$ and $p$ : carbon $2 p_{z}$. First row: "natural" ordering of the PAOs as suggested by the order of atoms along the chain. Second row: PAOs reordered on the basis of the exchange matrix elements $K_{i j}$.

$h_{1} h_{2} l_{3} l_{3} s_{3} l_{3} p_{3} h_{4} l_{5} p_{5} l_{5} s_{5} l_{5} h_{6} l_{7} l_{7} s_{7} l_{7} p_{7} h_{8} l_{9} p_{9} l_{9} s_{9} l_{9} h_{10}$ $s_{3} s_{5} h_{4} l_{5} h_{1} l_{3} l_{3} h_{2} p_{3} l_{3} l_{5} p_{5} l_{5} l_{7} p_{7} l_{7} l_{9} p_{9} h_{8} l_{9} l_{9} h_{10} l_{7} h_{6} s_{7} s_{9}$

pVTZ) basis ${ }^{33}$ by removing the $d$ function. The size of the PAO basis was 14 for $\mathrm{C}_{2} \mathrm{H}_{4}, 26$ for $\mathrm{C}_{4} \mathrm{H}_{6}$, and 38 for $\mathrm{C}_{6} \mathrm{H}_{8}$.

$\mathrm{Ab}$ initio calculations using restricted Hartree-Fock (RHF), MP2, and CCSD theory were performed with Q-CHEM in the PAO basis. (The reference used for the MP2 and CCSD wave functions was the RHF determinant of molecular orbitals in the PAO basis.) Corresponding groundstate energies are listed in Table II. Due to limitations in the integral-transformation code in Q-CHEM when using a large secondary basis, we were unable to obtain CCSD energies for $\mathrm{C}_{6} \mathrm{H}_{8}$.

Nonorthogonal orbital DMRG calculations with the nonHermitian algorithm, correlating all electrons in all the PAO orbitals, hereafter denoted as PAO-DMRG, were performed using the BLOCK code. ${ }^{9}$ In $\mathrm{C}_{2} \mathrm{H}_{4}$ and $\mathrm{C}_{4} \mathrm{H}_{6}$ we solved the Schrödinger equation using the iterative Davidson scheme, but for $\mathrm{C}_{6} \mathrm{H}_{8}$ we could not converge the Davidson algorithm even after hundreds of iterations, and thus exact diagonalization was used, limiting those calculations to $M=50$. Except where indicated, between eight and ten sweeps were carried out at each value of $M$. For these calculations, the PAOs were used in the order suggested by the linear chain. The specific ordering for $\mathrm{C}_{4} \mathrm{H}_{6}$ is given in Table I. Orderings of the PAOs in the other molecules are available as supplementary data.

In Table II, we compare the results obtained using PAODMRG for various sizes of subspace $M$ against the results of MP2 and CCSD calculations. The largest number of states retained, $M=300$, is smaller than what we have used in the past, reflecting the overhead of the non-Hermitian algorithm and our implementation. However, even at these modest $M$ values, the PAO-DMRG produces energies that are comparable to or better than the CCSD energies. CCSD accuracy is reached between $M=100$ and $M=200$ for $\mathrm{C}_{2} \mathrm{H}_{4}$ and roughly at $M=300$ for $\mathrm{C}_{4} \mathrm{H}_{6}$.

In a typical Hermitian DMRG calculation, for a fixed subspace size $M$, the finite sweep algorithm typically converges slowly when the remaining error in the sweep energy (due to not enough sweeps) is on the order of the error in the energy after the sweeps have converged, due to finite $M$. We observe a related phenomenon in the non-Hermitian calculations. Figure 1 shows the convergence of the non-Hermitian PAO-DMRG sweep energy as a function of the number of sweeps, keeping $M$ fixed at $M=100$, for $\mathrm{C}_{2} \mathrm{H}_{4}$. In the first few sweeps, the sweep energy converges rapidly, but then proceeds to oscillate, with a magnitude of oscillation $\sim 0.4 m E_{h}$, which is comparable to the magnitude of fixed $M=100$ error, estimated to be $\sim 1.1 m E_{h}$. Oscillations (as opposed to the monotonic convergence of the Hermitian algo-
TABLE II. DMRG, MP2, and CCSD energies in $E_{h}$ for the first three polyenes. PAO-DMRG: non-Hermitian DMRG in the PAO basis, MO-DMRG: Hermitian DMRG in the MO basis (MOs reordered using the $K$ matrix). $M$ is the number of retained states. Italics indicate that the digits are not fully converged with respect to the number of sweeps.

\begin{tabular}{|c|c|c|}
\hline \multicolumn{3}{|c|}{$\mathrm{C}_{2} \mathrm{H}_{4}$} \\
\hline HF -78.055 992 & MP2 -78.162 218 & CCSD -78.191812 \\
\hline M & PAO-DMRG & MO-DMRG \\
\hline 50 & -78.183 & -78.191 \\
\hline 100 & -78.1917 & -78.1920 \\
\hline 200 & -78.1926 & -78.1927 \\
\hline 300 & -78.19269 & -78.19275 \\
\hline \multicolumn{3}{|c|}{$\mathrm{C}_{4} \mathrm{H}_{6}$} \\
\hline HF -154.959490 & MP2 -155.166190 & CCSD - 155.217496 \\
\hline M & PAO-DMRG & MO-DMRG \\
\hline 50 & -155.167 & -155.187 \\
\hline 100 & -155.1835 & -155.2008 \\
\hline 200 & -155.2129 & -155.2059 \\
\hline 300 & -155.2174 & -155.2108 \\
\hline \multicolumn{3}{|c|}{$\mathrm{C}_{6} \mathrm{H}_{8}$} \\
\hline HF -231.860330 & MP2 -231.171310 & $\mathrm{CCSD}^{\mathrm{a}}$ \\
\hline M & PAO-DMRG & MO-DMRG \\
\hline 50 & -232.092 & -232.161 \\
\hline 100 & b & -232.1993 \\
\hline 200 & $\mathrm{~b}$ & -232.2117 \\
\hline 300 & $\mathrm{~b}$ & -232.2154 \\
\hline
\end{tabular}

$\overline{\mathrm{a}} \mathrm{Q}$-CHEM could not complete the calculation due to the cost of the four-index transformation of the secondary basis.

${ }^{\mathrm{b}}$ The Davidson iterations would not converge and exact diagonalization was too costly to complete the calculation.

rithm) are to be expected from a non-Hermitian algorithm, that is not bounded from below by a variational result. In Table II we report our DMRG energies with the converged digits; additional unconverged digits are given in italics.

Comparing the results from the different molecules, we see that the PAO-DMRG calculations are most accurate in ethene, but decrease in accuracy (relative to CC and MP2

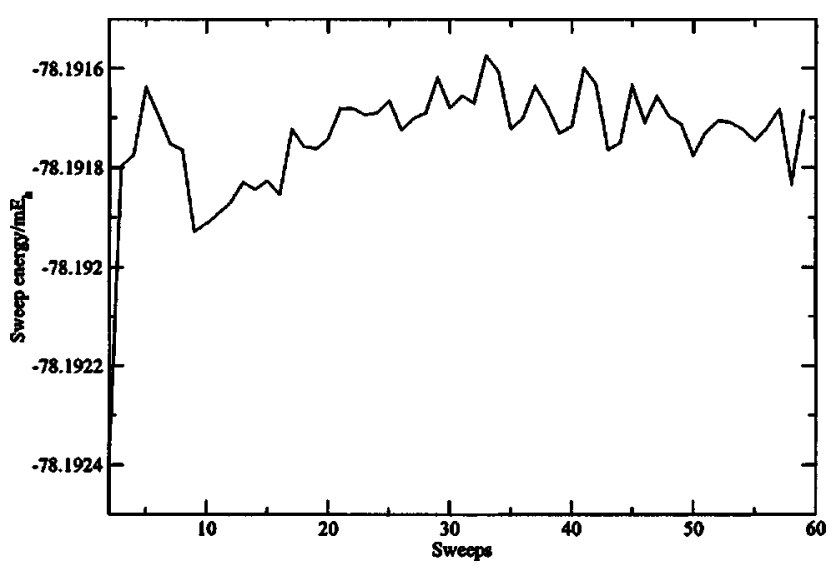

FIG. 1. Convergence of energies of successive sweeps in non-Hermitian PAO-DMRG calculations on $\mathrm{C}_{2} \mathrm{H}_{4}$ : rapid convergence is observed until the fifth iteration, after which the sweep energies exhibit small oscillations, as is expected in a non-Hermitian calculation. 


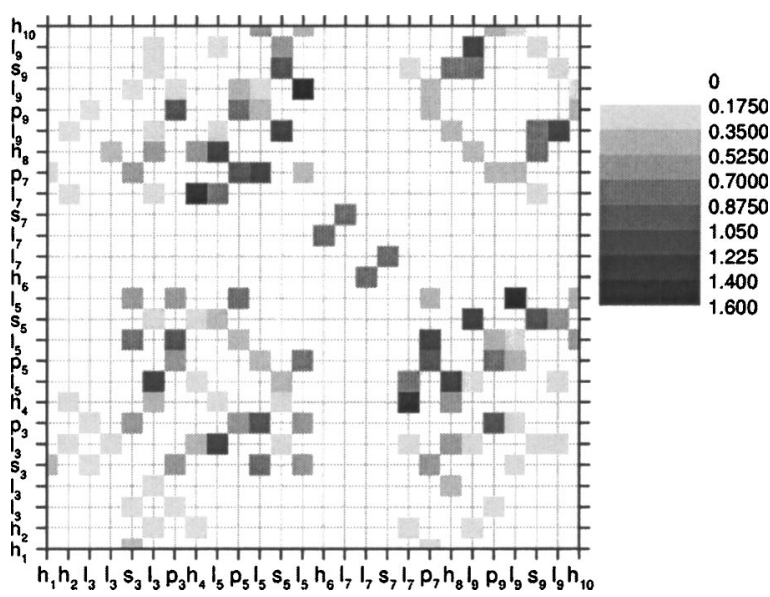

FIG. 2. The exchange matrix $K$ used for reordering the PAOs in $\mathrm{C}_{4} \mathrm{H}_{6}(|K|$ is plotted). Note that the diagonal $K_{i i}$, which is unimportant for the reordering, is set to zero. Atomic labels defined in Table I.

methods) for butadiene. Although the DMRG in a local basis should maintain a constant percentage accuracy for fixed $M$ for sufficiently long chains, as evidenced in numerous calculations on Pariser-Parr-Pople (PPP) Hamiltonians, ${ }^{34}$ we will need to go to longer chains than hexatriene to reach that scaling regime. In the PAO basis, there are five orbitals per $\mathrm{CH}_{2}$ unit. Taking $\mathrm{C}_{6} \mathrm{H}_{8}$ as an example, if we arrange the orbitals from each $\mathrm{CH}_{2}$ unit along one axis, and the successive $\mathrm{CH}_{2}$ units along another axis, the molecule looks like a $5 \times 6$ lattice of orbitals, which is not very one dimensional in its correlations at all. Consequently, we cannot really expect to reach the regime of constant $M$ until considerably longer chains, probably containing more than ten carbon atoms. Nonetheless, even in the short chain regime, the growth of the dimension of the DMRG subspace $\{S \circ\} \otimes\{E\}$ needed for a given energy accuracy, with chain length, is not too fast. For example, the number of CCSD amplitudes in $\mathrm{C}_{2} \mathrm{H}_{4}$ is $\sim 500$, which grows to $\sim 12000$ in $\mathrm{C}_{4} \mathrm{H}_{6}$; but the dimensions of the DMRG $\{S \circ\} \otimes\{E\}$ spaces required for roughly CCSD accuracy-corresponding to $M=100$ (in $\mathrm{C}_{2} \mathrm{H}_{4}$ ) and $M$ $=300\left(\mathrm{C}_{4} \mathrm{H}_{6}\right)$-are of dimensions $\sim 10000$ and $\sim 100000$, respectively, and thus grow more slowly than the number of CCSD amplitudes.

Given that these short chain polyenes are probably not very one dimensional in character, it is natural to consider different orderings of orbitals, as is commonly done in molecular DMRG calculations, ${ }^{8,14,35}$ so as to minimize correlations between widely spaced orbitals. We have chosen an ordering criterion recently advocated by Moritz et $a l^{35}$ based on minimizing the exchange integral $K_{i j}$ $=\iint r_{12}^{-1}\left|\phi_{i}\left(\mathbf{r}_{1}\right) \phi_{j}\left(\mathbf{r}_{2}\right)\right|^{2} d \mathbf{r}_{1} d \mathbf{r}_{2}$ between distant orbitals. The exchange matrix elements $K_{i j}$ for $\mathrm{C}_{4} \mathrm{H}_{6}$ are plotted in Fig. 2. Reordering $K_{i j}$ in $\mathrm{C}_{4} \mathrm{H}_{6}$ produced an ordering shown in the second row of Table I. General features of this reordering can be summarized as follows: (i) the core orbitals (which do not participate strongly in correlation) are moved to the ends of the chain, (ii) the carbon $\sigma$ and $\pi$ spaces are moved to the middle of the chain where they are treated more accurately, and (iii) the carbon $s p^{2}$ and hydrogen $1 s$ orbitals are paired together in clusters.
TABLE III. DMRG energies in $E_{h}$ for $\mathrm{C}_{4} \mathrm{H}_{6}$ using different orbitals and orderings. $M$ is the number of retained states. PAO (polarized atomic orbital) in natural ordering output by Q-CHEM (first ordering in Table I), MO (molecular orbitals, reordered on $K$ matrix), ord-PAO (PAOs reordered based on $K$ matrix), SO (symmetrically orthogonalized PAOs in natural ordering). Italics indicate that the digits are not fully converged with respect to the number of sweeps.

\begin{tabular}{rcccc}
\hline \hline $\mathrm{M}$ & PAO & MO & ord-PAO & SO \\
\hline 50 & -155.167 & -155.187 & -155.189 & -154.182 \\
100 & -155.1835 & -155.2008 & -155.2162 & -154.7404 \\
200 & -155.2129 & -155.2059 & -155.2185 & -155.1953 \\
300 & -155.2174 & -155.2107 & -155.2192 & -155.2146 \\
\hline \hline
\end{tabular}

We carried out DMRG calculations with the reordered set of PAOs for $\mathrm{C}_{4} \mathrm{H}_{6}$ and the energies are given in Table III. The new energies are indeed considerably improved relative to the PAO-DMRG results and already at $M=200$, the reordered DMRG energy is significantly better than the CCSD energy.

Finally, it is important to compare our PAO-DMRG calculations with Hermitian DMRG calculations in an orthogonal molecular-orbital (MO) basis to see what advantage has been gained over the standard DMRG treatment. We obtained MOs in the PAO basis, reordered them to minimize long range couplings in the exchange matrix $K$, and carried out standard Hermitian DMRG calculations, hereafter denoted as MO-DMRG. We should note that unlike in the nonHermitian DMRG calculations, no convergence difficulties in the Davidson algorithm were observed in any of the systems, although typically more Davidson iterations were needed for the longer chains due to the reduced separation between the ground and excited states. From the energies in Tables II and III, we see that compared to the PAO-DMRG results, the MO-DMRG energies are much better for small values of $M$ but less so, or even worse, for larger values of $M$. The better performance at small values of $M$ is due to the fact that the PAOs possess poor energy localization (i.e., they are not eigenfunctions of the Fock operator), and thus for small $M$, the DMRG tries first to "recorrelate" the PAOs back towards the MO basis. Such an orbital rotation may be considered a form of dynamical correlation, since it involves only low particle-number connected terms in a cluster expansion; consequently, such correlations are inefficiently captured by the DMRG in comparison with exponential ansatz methods such as CC theory. Eventually, however, the overall faster convergence of the DMRG energy with $M$ in the PAO basis as compared with the MO basis indicates that there are fewer long-range correlations in the PAO basis. This tradeoff between the effects of energy and space localization of the orbitals is expected to be a general feature in DMRG calculations on small molecules. In Table III we include results of DMRG calculations using a different local, symmetrically orthogonalized (SO) PAO basis (each orthogonal orbital $\eta_{i}^{\prime}$ is obtained from the corresponding PAOs via $\eta_{i}^{\prime}$ $=\Sigma_{j}\left[S^{-1 / 2}\right]_{i j} \eta_{j}$, where $S$ is the PAO overlap matrix). As expected, the behavior in this local basis compared to the MO basis is poor for the smaller $M$, but convergence with $M$ is rapid, and the SO-DMRG result is even better than the MO- 
DMRG result when $M=300$. In addition, this is consistent (as one expects) with the fact that the improved energies obtained with the PAO-DMRG algorithm as compared to the MO-DMRG algorithm are due to orbital spatial localization, and not due to the particular characteristics of nonorthogonality or the biorthogonal representation.

\section{CONCLUSIONS}

Here we have described the theory and implementation of two extensions of the density-matrix renormalizationgroup (DMRG) algorithm in quantum chemistry: (i) to use an underlying nonorthogonal one-particle basis, and (ii) to treat non-Hermitian and complex operators and complex wave functions. We handled the nonorthogonality of the oneparticle basis by first transforming into a biorthogonal representation. This led naturally to a non-Hermitian DMRG algorithm, which paralleled the usual Hermitian algorithm. There arose the problem of choosing an optimal density matrix in the non-Hermitian algorithm, and we showed that a truncation based on a function of the density-matrix operator representation in the biorthogonal space was the most natural.

We used these extensions to the DMRG algorithm to carry out preliminary calculations on the first three members of the polyene series in a nonorthogonal polarized atomicorbital (PAO) basis. Even retaining a small number of states ( $M$ values of only a few hundred), we could achieve accuracy comparable or better than coupled-cluster (CCSD) theory. In addition, DMRG calculations with localized PAOs (PAO-DMRG) were more accurate than in the MO basis (MO-DMRG), for larger values of the number of retained states $M$. This was due to the smaller number of long-range correlations in the localized PAO basis, leading to faster convergence with $M$. While similar benefits may be obtainable using orthogonal localized orbitals, we view allowing underlying nonorthogonality in the one-particle basis as adding additional flexibility to the DMRG algorithm.

Non-Hermiticity of the operators involved proved disadvantageous in several ways. First, the non-Hermitian DMRG algorithm was more expensive (eight times in compute cost, four times in memory cost) than the usual Hermitian DMRG algorithm. Second, the convergence of iterative diagonalization schemes employed to solve the Schrödinger equation in the DMRG subspace was very poor. While the extension of the DMRG to non-Hermitian Hamiltonians opens up new possibilities, e.g., to use similarity-transformed CC Hamilto- nians within the DMRG algorithm, the practicality of such methods will hinge on whether or not suitable preconditioners for the iterative diagonalization can be found.

\section{ACKNOWLEDGMENTS}

One of the authors (G.K.-L.C.) would like to acknowledge support from Cornell University. Another author (T.V.V.) would like to acknowledge support from MIT.

${ }^{1}$ S. R. White, Phys. Rev. Lett. 69, 2863 (1992).

${ }^{2}$ S. R. White, Phys. Rev. B 48, 10385 (1993).

${ }^{3}$ R. J. Harrison, J. Chem. Phys. 94, 5021 (1991).

${ }^{4}$ A. Auerbach, Contemporary Physics (Springer, New York, 1992).

${ }^{5}$ S. R. White and R. L. Martin, J. Chem. Phys. 110, 4127 (1998).

${ }^{6}$ A. O. Mitrushenkov, G. Fano, F. Ortolani, R. Linguerri, and P. Palmieri, J. Chem. Phys. 115, 6815 (2001).

${ }^{7}$ A. O. Mitrushenkov, R. Linguerri, P. Palmieri, and G. Fano, J. Chem. Phys. 119, 4148 (2003).

${ }^{8}$ G. K.-L. Chan and M. Head-Gordon, J. Chem. Phys. 116, 4462 (2002).

${ }^{9}$ G. K.-L. Chan, J. Chem. Phys. 120, 3172 (2004).

${ }^{10}$ G. K.-L. Chan and M. Head-Gordon, J. Chem. Phys. 118, 8551 (2003).

${ }^{11}$ G. K.-L. Chan, M. Kállay, and J. Gauss, J. Chem. Phys. 121, 6110 (2004).

${ }^{12}$ Ö. Legeza, J. Roder, and B. A. Hess, Mol. Phys. 101, 2019 (2003).

${ }^{13}$ Ö. Legeza, J. Roder, and B. A. Hess, Phys. Rev. B 67, 125114 (2003).

${ }^{14}$ Ö. Legeza and J. Solyom, Phys. Rev. B 68, 195116 (2003).

${ }^{15}$ G. E. Scuseria and P. Y. Ayala, J. Chem. Phys. 111, 8330 (1999).

${ }^{16}$ P. E. Maslen and M. Head-Gordon, Chem. Phys. Lett. 283, 102 (1998).

${ }^{17}$ A. O. Mitrushenkov, G. Fano, R. Linguerri, and P. Palmieri, e-print condmat/0306058.

${ }^{18}$ W. Hofstetter, I. Affleck, D. Nelson, and U. Schollwöck, Europhys. Lett. 66, 178 (2004).

${ }^{19}$ X. Wang and T. Xiang, Phys. Rev. B 56, 5061 (1997).

${ }^{20}$ T. Enss and U. Schollwöck, J. Phys. A 34, 7769 (2001).

${ }^{21}$ S. R. White, J. Chem. Phys. 117, 7472 (2002).

${ }^{22}$ X. Li and J. Paldus, J. Chem. Phys. 113, 9966 (2000).

${ }^{23}$ M. S. Lee and M. Head-Gordon, J. Chem. Phys. 107, 9085 (1997).

${ }^{24}$ M. Head-Gordon, P. E. Maslen, and C. A. White, J. Chem. Phys. 108, 616 (1998).

${ }^{25}$ E. R. Davidson, J. Comput. Phys. 17, 87 (1975).

${ }^{26}$ in Templates for the Solution of Algebraic Eigenvalue Problems: A Practical Guide, edited by Z. Bai, J. Demmel, J. Dongarra, A. Ruhe, and H. van der Vorst (SIAM, Philadelphia, 2000).

${ }^{27}$ J. Olsen, P. Jorgensen, and J. Simons, Chem. Phys. Lett. 169, 463 (1990).

${ }^{28}$ K. Nakayama, H. Nakano, and K. Hirao, Int. J. Quantum Chem. 66, 157 (1999).

${ }^{29}$ R. J. Cave and E. R. Davidson, J. Phys. Chem. 91, 4481 (1987).

${ }^{30}$ G. Berghold, M. Parrinello, and J. Hutter, J. Chem. Phys. 116, 1800 (2002).

${ }^{31}$ J. P. Foster and F. Weinhold, J. Am. Chem. Soc. 102, 7211 (1980).

${ }^{32}$ J. Kong, C. A. White, A. I. Krylov et al., J. Comput. Chem. 21, 1532 (2000).

${ }^{33}$ T. H. Dunning, Jr., J. Chem. Phys. 90, 1007 (1989).

${ }^{34}$ R. J. Bursill and W. Barford, Phys. Rev. B 66, 205112 (2002).

${ }^{35}$ G. Moritz, B. A. Hess, and M. Reiher, J. Chem. Phys. 122, 024107 (2005). 